\title{
Factorial Validity Structure of Occupational Stress
}

\author{
Cruz García Lirios* \\ Autonomous University of Mexico State, UAEMEX, Mexico \\ *Corresponding author: Cruz García Lirios, Autonomous University of Mexico State, UAEMEX, Mexico
}

\section{ARTICLE INFO}

Received: August 02, 2020

Published: 幽 August 14, 2020

Citation: Cruz García Lirios. Factorial Validity Structure of Occupational Stress. Biomed J Sci \& Tech Res 29(4)-2020. BJSTR. MS.ID.004823.

\section{ABSTRACT}

The objective of the present work was to specify a model for the study of work stress, considering a review of the literature that emphasizes three components related to exhaustion, neglect, and frustration. A non-experimental, cross-sectional, and correlational study was carried out with a selection of 100 workers from a public hospital, considering their working hours, as well as their seniority. A structural equation model was established in which exhaustion was the hegemonic component that explained the highest percentage of variance with $27 \%$, although the research design limited the results to the research scenario, suggesting its extension to another. context.
\end{abstract}

Keywords: Work Stress; Emotional Exhaustion; Job Depersonalization and Professional Frustration

\section{Introduction}

Work stress understood as the somatization of an occupational disease and indicated by high levels of exhaustion and resistance, negligence and commitment, as well as frustration and violence has been a central theme and theme of the organizational agenda [1]. Work stress understood as the somatization of an occupational disease and indicated by high levels of exhaustion and resistance, negligence and commitment, as well as frustration and violence has been a central theme and theme of the organizational agenda [2]. It is an adaptive syndrome and it develops in three phases;

1. Resistance to the increase in demands and the reduction of psychological and organizational resources,

2. Alarm or intensification of the work rhythm, as well as a disproportionate increase in objectives, tasks and goals,

3. Exhaustion or maximization of individual functions and resources, as well as low performance and increased errors [3].

Since the work stress process is a complex problem, this work approaches it from a theoretical approach of its components, emphasizing the link with organizations and their central actors such as leaders, talents, peers and operatives [4]. Next, the models and instruments that measure occupational stress are addressed, highlighting the environment, the individual and the organization as central axes of reducing demands and increasing resources to prevent the syndrome [5].

\section{Theory of Occupational Stress}

Productive and health care organizations are prone to develop a stress structure or, in the opposite case, a happiness structure, both derived from a structure of group dynamics, organizational climate and conflicts. homework and relationships [6]. Done and hospitals in both entities' prevention of diseases and attention to health, stressful relationships develop between their representatives and employees [7]. Psychological studies around stress have shown the existence of a three-dimensional structure in which three factors converge: emotional exhaustion, job depersonalization and professional frustration. These investigations shown that stress is influenced by interpersonal and intergroup relations. In this sense, group dynamics has been identified as the main mechanism of influence that, from conflicts, modifies the work structure of an organization [8].

In the case of stress, relationship conflicts and task conflicts are the main causes of emotional exhaustion and job depersonalization. Some studies show that the conflict in relationships influences this last (García, 2020d). Others argue that organizational climate would not have a significant effect on emotional exhaustion or in 
professional frustration [9]. However, it is evident that the factors that make up a structure of job happiness are determinants of professional frustration [10]. Such causal variables are dedication, enjoyment, and work vigor [11]. As happiness factors at work increase, professional frustration decreases. However, these studies show causal relationships between factors without having demonstrated their structure [2]. Therefore, this research is to goose demonstrate the three - dimensional structure supported by the theory of job stress [12].

The theory of work stress raises three explanatory dimensions of exhaustion, frustration and neglect observed in health and education professionals predominantly in their occupational field [13]. These are three components that by themselves suggest a prevalence of stress, although together they denote a mental illness known as the syndrome of overwork and which is indicated by high levels of conflict within the organization [14]. It is assumed that in occupational settings, since occupational health is focused on people rather than organizations, there are signs of exhaustion, as would be the case of those who carry out exhaustive work based on their degree of competence, relationship with superiors or peers [15]. These types of workers suffer from exhaustion due to their function, although as they gather the merits to perform another function they continue to act with occupational sacrifice, assuming that their merits are not enough [16]. It is a very common condition in vertical organizations, focused on traditional leadership, unilateral communication and normative motivation [17].

Negligence is often assimilated as a strategy or response to the increase in an increasingly expensive and complex labor demand in terms of effort and cooperation, being deferred to the person least capable or motivated to carry out the management, production or translation. of homework [18]. The developing syndrome is often indicated by solipsistic communication, self-centered motivation, and absence of goals and objectives, always attributable to the organization, leaders, or peers [19]. The most significant component of the work stress syndrome is the frustration that when associated with violence is a pending issue for organizations [20]. It is a common problem in companies dedicated to the efficiency, effectiveness and effectiveness of their processes and products, such as optimization and innovation [18]. The condition develops and develops in talents rather than leaders or peers, as well as other types of logistics or operational personnel [21]. Studies of work stress and systematic reviews of occupational health have shown the prevalence of these three factors, as well as the hegemony of exhaustion as a visible factor in the syndrome of overwork associated with occupational diseases and accidents [22].

\section{Studies of Occupational Stress}

In the framework of the strategic alliances between organizations and institutions for the labor insertion of talents, the models and instruments that measure the problem have focused their attention on the skills determined by self-confidence, selfefficacy and self-esteem [23]. The Stress Control Scale states that the worry of error and the somatization of anxiety are determinants of skills and is in the prevention of an accident [24]. The Perceived Stress Scale includes reagents alluding to stressors; assessment of the situation, resources and environment, as well as emotional and behavioral responses, associated with sociodemographic variables as determinants of the physiology of somatization of disease, although it only refers to working hypotheses to be tested in differential situations of resources and demands [25]. Both models generalize their relationships from considering that the demands of the environment affect the resources of organizations, including the psychological resources of those who suffer from stress [26].

In a more specific sense and continuing with this principle of external influence on biomedical aspects [27]. The Psychological Stress Scale sets out to differentiate chronic and acute levels to establish its relationship with the metabolic syndrome, indicated by the waist circumference, and the level of trigricerides, cholesterol, glucose and pressure [28]. This biomedical model links the internal factors of the individual with molecular biomedical aspects [29]. The three models with their corresponding instruments seem to demonstrate that the work environment even affects biomedical aspects that would indicate a level of resistance, alarm or exhaustion [30]. Consequently, the measurement of factors related to this process of internalization of demands and externalization of effects involves at least three components that would make up a robust structure [31].

\section{Method}

Sample. He interviewed or 100 employees $(M=35,4 S D=2,3$ and $M=1^{\prime} 324,31$ SD $=243,56$ USD) of the hospital General of the city of Cuernavaca, Morelos (Mexico).

\section{Instrument}

Is utilize the Scale Job Stress, that measurement exhaustion, depersonalization and frustration of staff. It includes 138 items, with four response options ranging from $0=$ "strongly disagree" to $5=$ "strongly agree".

\section{Procedure}

Respondents were informed that the results of the study would not positively or negatively affect their contractual employment status with the institutes where they work. Once solved the questionnaire, the trend of answers and verified, in cases where its response or he focused on a choice, he asked the participants to write on the back the reasons for their decisions. The data was processed in the SPSS and LISREL software, in their student versions [32-41]. 


\section{Results}

A normality analysis was performed to establish the distribution of the answers to the questions and questions of the instrument. The selection criteria included the values that are between -3 to +3 . The items and questions excluded for exceeding the allowable range. Subsequently, exploratory factor analysis of main components with varimax rotation to corroborate the three - dimensional structure. The selection criteria reagent was configured factors higher correlation 0300 between each reagent and the appropriate factor. The first factor, referred to emotional exhaustion, explained $27 \%$ of the variance; the second, related to labor depersonalization, 7\%, and the third, which refers to the frustration staff, the $5 \%$. Finally, the reliability of the underlying factors was established. The values of normality and reliability of the subscales that measure the factors found. The factor of emotional exhaustion scored low reliability, which means the heterogeneity of results in relation to the systematization of interviews with the same subjects. In contrast, the factors that allude to job depersonalization, professional frustration and work stress obtained enough reliability values.

Once the normality, validity and reliability of the subscales were demonstrated, a correlation was carried out between the factors to establish their direct and significant associative relationships. It shows three significant associations between the factors. Only in the established one between professional frustration and depersonalization is there a negative relationship. In other words, as the lack of achievement increases, personalized attention increases. In this sense, the lack of socialization is to link with stress at work. Finally, another contributing factor to stress is lack of achievement. Associative relationships are preliminary to causal relationships. Therefore, a successive step multiple linear regression analysis was performed to establish the main effect of the exposed factors. The causal relationships between the factors. From the successive step's technique, it was established that the factor corresponding to personal frustration has a direct positive and significant effect on work stress. In a second moment, the factor that alludes to personal depersonalization turned out to be the determining variable in work stress. In this sense, the negative and significant association between the factors suggests the absence of effects of variables not included in the model. However, to demonstrate non-collinearity, a covariance analysis was performed.

The analysis of covariances was carried out with the LISREL software. Consider is to $r$ on the "phi" parameters and "zeta" to establish the absence or presence of collinear relationship and the effect of other variables inferred by the level of disturbance. Twodimensional structure of work stress. The covariance parameter "phi" is very high, which means that the effect of other variables not included in the model is minimal and with it the probabilities of collinearity. However, it is noted that the disturbance parameter is very high, evidenced in the incidence of other variables. In order to observe the structure of axes, trajectories and relationships between the three established factors and their indicators, we proceeded to estimate a model of structural equations.

A structure of reflective relationships is observed between the factors with respect to their indicators, as well as the prevalence of the total variance explained by the exhaustion factor. The adjustment parameters $\Upsilon_{\chi=24,3}(12 \mathrm{df}) \mathrm{p}>, 05 ; \mathrm{GFI}=, 997$; CFI = ,995; RMSEA $=, 007 J$ suggest the non-rejection of the hypothesis regarding the theoretical relationships reported in the literature regarding the findings observed in the present work.

\section{Discussion}

The contribution of this work to the state of knowledge lies in the establishment of an exploratory factor structure as evidence of validity and reliability of the instrument that measures the axes, trajectories, and relationships between exhaustion, neglect, and frustration proposed in the work stress model. However, the research design has limitations regarding the findings, which are not generalizable and are confined to the surveyed sample, as well as the suggestion to extend the work to other samples in order to contribute to the validity of the instrument. In relation to the models and instruments reviewed, the present work notes the same prevalence of the exhaustion factor, although the validity is less than that reported by the other studies. The construction of an instrument with greater reliability and validity will allow us to discuss the scope of this phenomenon as part of occupational health in the face of contingencies in which demands are exacerbated and resources are scarce. Thus, with regard to the work environment as a determinant of the asymmetries between demands and resources, as well as its effects on the syndrome of overwork, it is necessary to establish the factors that most influence occupational health and its consequences on stress and The depletion.

In the case of organizationalvariables such as work environment, training and training centered on objectives, tasks and goals, indicators of job demand, this study indicates that its effects must be observed in exhaustion. As goals become more complex, tasks are intensified, and goals are prolonged, work-related stress seems to fall on the exhaustion of those who are responsible for carrying out the strategies and tasks to achieve these ends. Regarding the effects of stress and exhaustion on performance, competitiveness and innovation, this study has shown a $27 \%$ variance explained by this factor, which suggests the inclusion of other factors such as neglect or frustration. Research lines concerning external factors as determinants of other variables concomitant to exhaustion will allow us to explain occupational risks and their effects on occupational health. 


\section{Conclusion}

The structure of work stress is two-dimensional. Professional frustration and job depersonalization are the main determining factors. Although first is the leading cause of work -related stress, the effect of other variables is evident, including: the exhaustion emotional in the case of stress ; he dedication, enjoyment and vigor at work in the case of happiness at work ; and leadership, task conflicts and relationships in the case of group dynamics at work. In other words, under a stressful structure a structure of individual happiness and group dynamics could underlie. The influence of structural factors in each of them could demonstrate the ambivalence that characterizes productive organizations.

\section{References}

1. García C (2020) Model of intangible assets and capitals in organizations. Journal of Neurology Psychiatry and Brain Research 1(1): 1-9.

2. Garcia C (2019) Administration of social work model for local cooperativism. Journal of Strategy Management 8(2): 35-48

3. Adams S (2020) Academic framework of social entrepreneurship. International Journal of Research Aspects of Engineering \& Management 16(2): 1-5.

4. García C (2020) Reliability and validity of an instrument that measures corporate social responsibility. Social Science \& Humanities Journal 4(2): 1781-1789.

5. Molina MR (2020) Exploratory algorithmic factorial structure of occupational health. Advanced Research Journal of Multidisciplinary Discoveries 10(5): 40-50.

6. García C (2020) Specification a model for study of entrepreneurship. Advanced Research Journal of Multidisciplinary Discoveries 49(1): 1-4.

7. Juárez M (2020) Specification a model for study of corporate assistance. Global Journal of Archeology \& Anthropology 11(2): 50-54.

8. Korstanje M (2020) Academic framework of knowledge management International Journal of Engineering Technology and Management Research 7(2): 1-6.

9. Elizarraráz G (2020) Metanalytical validity of the technology utility perception scale. International Journal of Psychiatry Research 3(8): 1-7.

10. García C (2020) Specification a model for study of knowledge management. International Journal of Neurobiology 2(1): 1-3.

11. Quiroz CY (2020) Specification a model of digital entrepreneurship. Current Research in Psychology \& Behavioral Science 1(1): 1-4

12. Bermudez G (2019) Meta-analytical validity of the social entrepreneurship inventory: a study of random effects size. Global Journal of Management \& Business Research 19(10): 15-19.

13. Carreon J (2019) Categorical exploratory structure of intellectual capita formation in its phase of intangible organizational assets. Journal of Social Science Research 6(8): 1-6.

14. García C (2019) Exploratory dimensions of the attitude toward occupational health. Entrepreneurship Dimension 7(3): 1-8.

15. Aguilar JA (2019) Specification a model for study of utility perception. Journal of Communication \& Health 9 (2): 47-54.

16. García C (2019) Exploratory factor structure of professional training expectations. 25(32): 252-270.

17. Carreon J (2019) Exploratory categorical structure of employment expectations. Journal of Social Science Research 6(8): 1-6.
18. Carreon J (2019) Model of fixed effects of diffuse variables in the formation of intellectual capital. International Journal of Engineering Research \& Development 15(19): 1-7.

19. Hernandez J (2019) Exploratory factor structure of well-being. Applied Environment Research 6(1): 1-5.

20. García C (2020) Specification a model for study of knowledge management. International Journal of Neurobiology 2(1): 1-3.

21. Elizarraráz G (2020) Metanalytical validity of the technology utility perception scale. International Journal of Psychiatry Research 3(8): 1-7.

22. García C (2020) Specification a model for study of occupational health. Global Journal of Management and Business Research 20(1): 1-6.

23. García C (2020) Specification a model of community health. Global Journal of Addiction \& rehabilitation Medicine 6(5): 63-66.

24.Carreon J (2019) Model of the determinants of human capital. International Journal of Advances in Social Science \& Humanities 7(8): $1-5$

25. Garcia C (2019) Specification a model for study of alocal entrepreneurship model. Earth \& Environmental Science Research \& Review 2(5): 1-3.

26. Martínez E (2019) Hybrid determinant model of the coffee entrepreneurship. Interconnecting 4(8): 111-143.

27. García C (2019) Specification a model for study of local development. Saudi Journal of Business \& Management Studies 4(11): 1-4.

28. García C (2019) Specification a model for study of quality of life governance. Saudi Journal of Business \& Management Studies 4(2): 1-4.

29. Sanchez A (2019) Specification a model for the study of management culture. Spirals 3(31): 1-11.

30. Hernandez TJ (2019) Specification a model for study of vocational training. Journal Education \& Social Policy 6(2): 1-4.

31. Garcia C (2019) Specification a model for study of social entrepreneurship. Global Advanced Research Journal of Medicine \& Medical Science 8(1): $1-4$

32. García C (2019) Specification of a self-care model. Lux Medica 42(1): $15-25$.

33. García C (2020) Model of intangible assets and capitals in organizations. Journal of Neurology Psychiatry and Brain Research 1(1): 1-9.

34. Aguilar JA (2019) Specification a model for study of utility perception. Journal of Communication \& Health 9 (2): 47-54.

35. Bermudez G (2019) Meta-analytical validity of the social entrepreneurship inventory: a study of random effects size. Global Journal of Management \& Business Research 19(10): 15-19.

36. Carreon J (2019) Model of the determinants of human capital. International Journal of Advances in Social Science \& Humanities 7(8): $1-5$

37. Carreon J (2019) Specification of a local entrepreneurship model. Saudi Journal of Business and Management Studies 4(11): 856-859.

38. Espinoza F (2019) Governance of migratory flows from establishment of identity and agenda of occupational health. Migration 4(7): 139-171.

39. Martínez E (2019) Hybrid determinant model of the coffee entrepreneurship. Interconnecting 4(8): 111-143.

40. Martinez E (2019) Model of the determinants of vocational training. International Journal of Advances in Social Science \& Humanities 6(7): $1-5$

41. Quiroz CY (2019) Specification a model of culture knowledge. Global Advanced Research Journal of Agricultural Science 8(10): 1-4. 
ISSN: 2574-1241

DOI: 10.26717/BJSTR.2020.29.004823

Cruz García Lirios. Biomed J Sci \& Tech Res

(C) (P) This work is licensed under Creative Commons Attribution 4.0 License

Submission Link: https://biomedres.us/submit-manuscript.php

$\begin{array}{ll}\text { BIOMEDICAL } & \text { Assets of Publishing with us } \\ \text { RESEARCHES } & \text { - Global archiving of articles } \\ \text { - Immediate, unrestricted online access }\end{array}$

